- Case Report

\title{
A Case Report of Steroid Withdrawal Syndrome
}

\author{
Faziana Wahab, Razlina Abdul Rahman*, Lili Husniati Yaacob, Norhayati Mohd Noor, Nani Draman \\ Klinik Rawatan Keluarga, Hospital Universiti Sains Malaysia, Kubang Kerian, Malaysia
}

We report a case of steroid withdrawal syndrome in a 74-year-old woman who was suspected of having an occult exogenous Cushing's syndrome secondary to prolonged traditional complementary medicine use. She presented with non-specific symptoms of lethargy, malaise, and poor oral intake with weight loss for 1 month, and investigations showed suboptimal $9 \mathrm{AM}$ cortisol level. She has responded well to steroid replacement.

Keywords: Steroid; Cushing Syndrome; Traditional Medicine 


\section{INTRODUCTION}

The use of traditional complementary medicine (TCM) is common in Malaysia, particularly in a community setting. More than half of the patients have used at least one type of traditional medicine, and the most frequently used is herbal medicine. ${ }^{1,2)}$ Studies have shown that steroids are contained in more than half of the traditional medicine products that have been tested, and most of them were not registered. ${ }^{3)}$ Traditional medications that are contaminated with steroids can cause conditions, such as steroid withdrawal syndrome, especially if taken for a long period. ${ }^{3)}$ Steroid withdrawal syndrome occurs when there is an abrupt cessation or reduction of steroids in patients who take a supraphysiologic dose of steroid over a long period of time. ${ }^{4)}$ The symptoms are similar to adrenal insufficiency, but the patient's serum cortisol level is suboptimal. Although the symptoms are self-limiting, doctors need to be aware of this condition as steroid replacement may alleviate the symptoms and improve the patient's quality of life. ${ }^{4)}$

\section{CASE REPORT}

A 74-year-old woman with underlying symptoms of hypertension, hyperlipidemia, diabetes mellitus, post-thyroidectomy hypothyroid, and bilateral knee osteoarthritis presented with poor oral intake and severe lethargy for 1 month. She had reduced appetite that was associated with occasional nausea and vomiting. She experienced extreme lethargy that severely impaired her mobility, which required her to use a wheelchair. Although she experienced weight loss, the amount of weight loss could not be quantified by her. There were no hypothyroid symptoms and no other systemic symptoms. Despite visiting several general practitioners, her condition did not improve. The patient was on felodipine (10 mg oral dose [OD]), atorvastatin (40 mg OD), metformin-XR (750 mg OD), and L-thyroxine (50 mcg OD). She complied

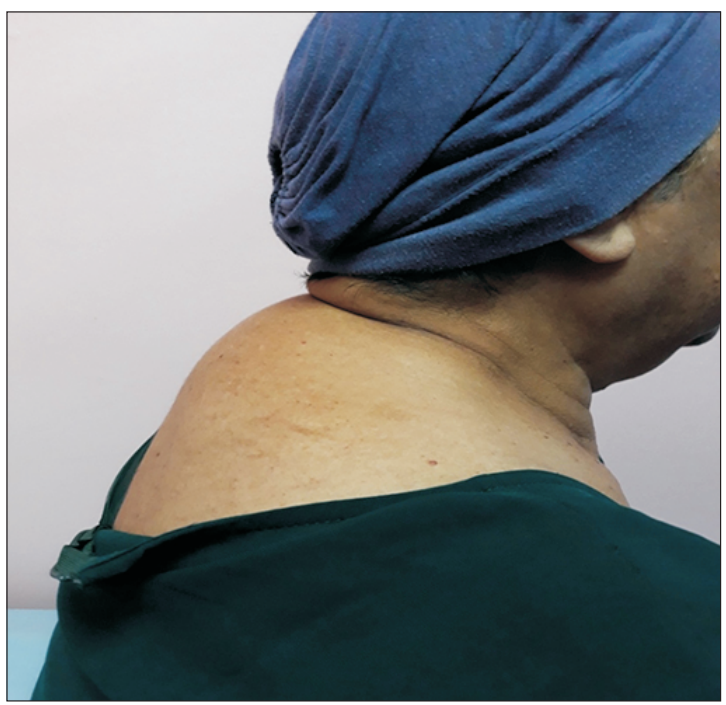

Figure 1. Dorsocervical fat pad (buffalo hump). Written informed consent for publication of this image was obtained from the patient. with her medications except for an oral hypoglycemic agent since her oral intake was reduced. On further questioning, the patient admitted to a history of prolonged TCM use. She had been taking a type of herbal medication known as "majun" for the past 2.5 years. Maajun was taken for general well-being and to help alleviate her knee pain secondary to osteoarthritis. She decided to stop taking the medication after being advised by her daughters. She noticed that the symptoms developed 1 week after she stopped taking the herbal preparation.

On examination, she had cushingoid features (Figures 1-4) such as moon facies, truncal obesity, buffalo hump, thin skin, and hirsutism. All vital signs and systemic examinations were normal except for random capillary blood sugar ( $4.4 \mathrm{mmol} / \mathrm{L})$, which was low. Blood investigations results including full blood count, renal function and thyroid function test were within the normal range except for the presence of

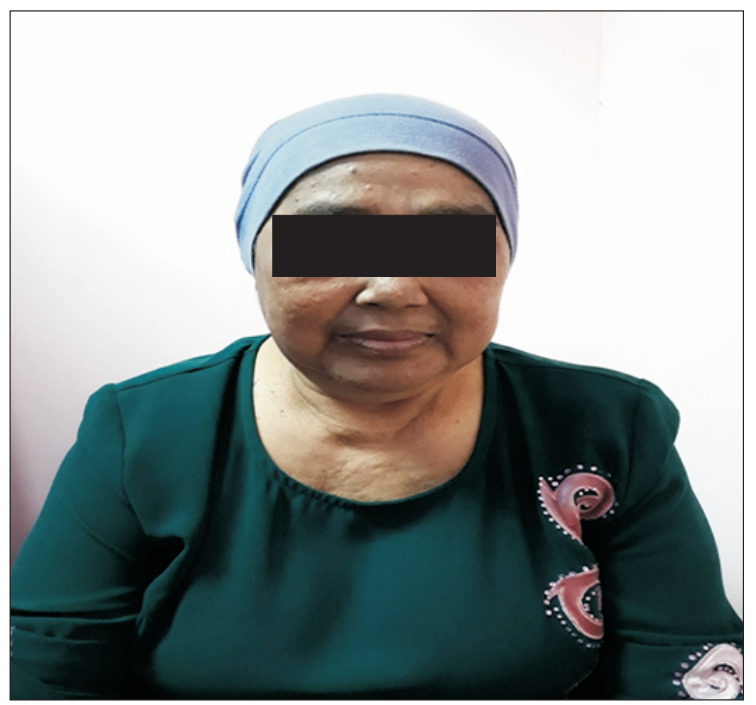

Figure 2. Moon face. Written informed consent for publication of this image was obtained from the patient.

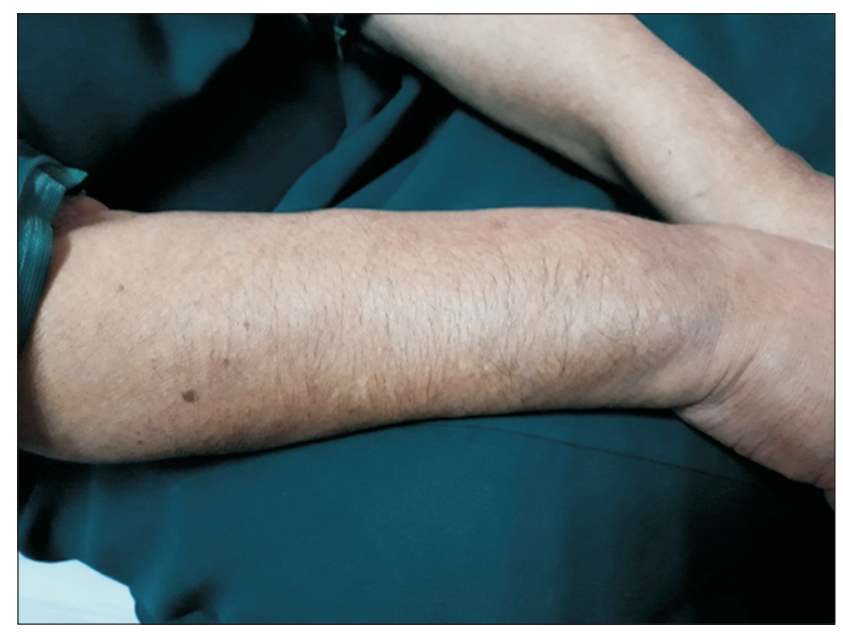

Figure 3. Excessive hairiness. Written informed consent for publication of this image was obtained from the patient. 


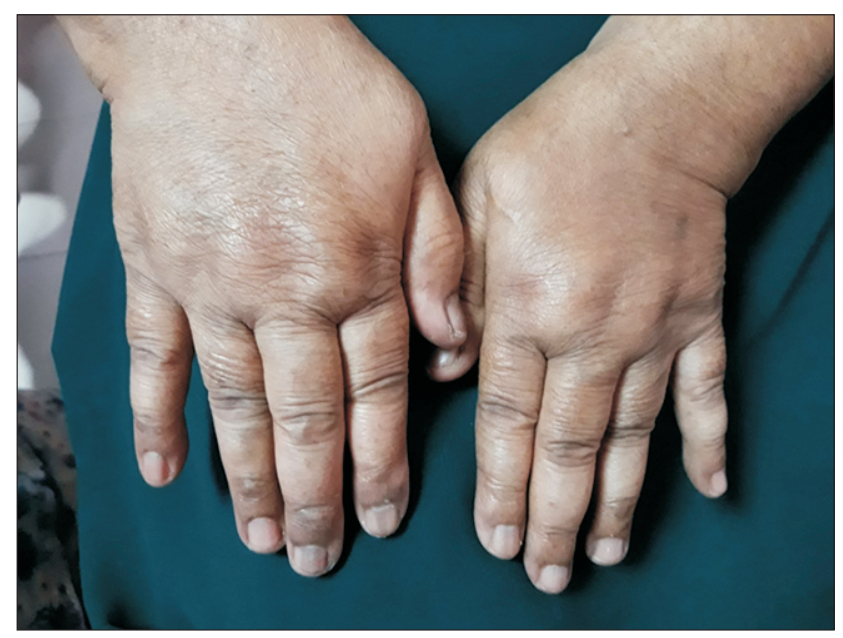

Figure 4. Thin and dry skin. Written informed consent for publication of this image was obtained from the patient.

mild transaminitis, an aspartate aminotransferase of $66 \mathrm{U} / \mathrm{L}$ (normal range, 5-34 U/L), and an alanine aminotransferase of $50 \mathrm{U} / \mathrm{L}$ (normal range, $<34 \mathrm{U} / \mathrm{L})$. Her diabetes was also fairly controlled with hemoglobin Alc (HbAlc) of $6.8 \%$.

Morning serum cortisol was measured to rule out steroid withdrawal syndrome based on the patient's history and physical findings. Serum cortisol was within the normal range at $193.1 \mathrm{nmol} / \mathrm{L}$ (normal range, $166-507 \mathrm{nmol} / \mathrm{L}$ ). The patient was then treated for steroid withdrawal syndrome. Mild transaminitis could have been caused by hepatotoxicity induced by TCM use.

She was started on tablet prednisolone (10 mg OD) and was on tapering doses for 6 weeks. The patient showed significant improvement in clinical symptoms after 2 weeks of low-dose steroid, and there was recurrence in the symptoms after prednisolone was stopped. An oral hypoglycemic agent was also stopped since her repeated HbAlc was $6.4 \%$ with normal blood glucose levels. Liver transaminase levels was also within a normal range at follow-up.

Written informed consent was obtained from the patient for publication of this case report and accompanying images. A copy of the written consent is available for review by the editor of this journal.

\section{DISCUSSION}

Maajun is one of the most common herbal medicines used by the Malay population. Usually it is produced from various tree roots or herbs and is processed into pills or paste. Furthermore, sometimes these traditional medicines are mixed with toxic heavy metals or undeclared drugs. ${ }^{5)}$ In 2005, half of the traditional medicine products that had been tested contained steroids, and most of them were not registered. ${ }^{3)}$ However, in our case, the patient refused to give a sample of her herbal preparation; therefore, we were unable to confirm the presence of steroids in her herbal medication.

Nevertheless, based on her history and physical examination, we believed that the medication used contained steroids, thus causing exogenous Cushing's syndrome secondary to prolonged TCM use. Several similar cases have also been previously reported ${ }^{6-8)}$ It is very likely that her diabetes mellitus was also induced by the prolonged use of the TCM; she was diagnosed with diabetes after using maajun for more than a year. This is supported by the observation that her blood sugar level normalized after stopping the usage of maajun.

People with prolonged exogenous exposure to steroids will experience a suppression of the hypothalamic-pituitary-adrena (HPA) axis. A sudden withdrawal of the exogenous supply of steroids will result in symptoms similar to adrenal insufficiency since the adrenal gland will not have enough time to produce hormones to meet the body's requirement. In patients with adrenal insufficiency, evidence of suppressed HPA axis are observed. ${ }^{9)}$ However, if there is no evidence of this, the patient is considered to have steroid withdrawal syndrome. ${ }^{9)}$ This case is a typical example of steroid withdrawal syndrome where she presented with the symptoms after she stopped taking the traditional medication.

Steroid withdrawal syndrome is defined by symptoms of glucocorticoid deficiency such as general malaise, lethargy, postural dizziness, generalized weakness, arthralgia, headaches, mood swings, and emotional labiality, in the setting of a proven normal HPA axis. ${ }^{10)}$ The integrity of the HPA axis can be assessed by measuring the 9 AM serum cortisol level. ${ }^{10)}$ A value $<100 \mathrm{nmol} / \mathrm{L}$ is suggestive of cortisol insufficiency whereas a value $>450 \mathrm{nmol} / \mathrm{L}$ is consistent with cortisol sufficiency. ${ }^{10)}$ A suboptimal value of $9 \mathrm{AM}$ serum cortisol level is defined as a value between 100 to $450 \mathrm{nmol} / \mathrm{L}$, and usually a patient with steroid withdrawal syndrome has a suboptimal value of 9 AM serum cortisol. ${ }^{4)}$ For further confirmation of adrenal insufficiency, stimulation tests such as the short synacthen test, glucagon stimulation test, or insulin tolerance test can be performed. ${ }^{10)}$ In this patient, however, we did not proceed with the stimulation test since her history was very suggestive and due to the lack of resources. Furthermore, the patient's symptoms improved after steroid replacement.

Current literature supports the reinstitution of steroids as treatment of steroid withdrawal syndrome. To withdraw steroids in a patient who is chronically taking exogenous steroids, the steroids should be gradually reduced to physiological doses (usually 5-7.5 mg of prednisolone) and then reduced by $1 \mathrm{mg}$ fortnightly to $1 \mathrm{mg}$, and then $1 \mathrm{mg}$ every other day for 14 days. ${ }^{10)}$ If the patient develops steroid withdrawal symptoms with a suboptimal 9 AM serum cortisol level, a longer duration for reduction of steroids is needed, which is then stopped once the symptoms are resolved. ${ }^{10)}$ However, there are no clear guidelines on how to replace steroids in patients where the amount of exogenous steroid taken is unknown. In this case, we had difficulty in deciding the replacement dose since we were not privy to the amount taken before. The fact that the patient had stopped the maajun for 2 weeks prior to her presentation makes it more difficult to gauge the starting dose. Nevertheless, due to her age and other comorbidities, we decided to start her on a short course of low-dose prednisolone with plans to taper the dose. 
Although the use of TCM is very prevalent in the Malay community, few cases are presented with a clear history as we have illustrated here. Most patients would have developed the complications insidiously and may not have connected it to the use of TCM. This is usually the case when the presentations are vague, which may masquerade as other illnesses. This fact highlights the importance for a primary care practitioner to be alert and vigilant to the possibilities and to be suspicious of patients, especially in those suffering from chronic illnesses that may expose them to the use of occult steroids.

In conclusion, since usage of TCM may be common among patients, especially in those with chronic illnesses, physicians in a primary care setting should be aware of occult usage of steroids in these patients. Thus, any complications from chronic steroid usage should be assessed in these patients. Additionally, the physicians should inform patients regarding the harmful effects of TCM.

\section{CONFLICT OF INTEREST}

No potential conflict of interest relevant to this article was reported.

\section{ORCID}

Faziana Wahab: https://orcid.org/0000-0002-3951-5365

Razlina Abdul Rahman: https://orcid.org/0000-0003-2593-1832

Lili Husniati Yaacob: https://orcid.org/0000-0003-1296-0482

Norhayati Mohd Noor: https://orcid.org/0000-0002-6372-1476

Nani Draman: https://orcid.org/0000-0003-4143-6065

\section{REFERENCES}

1. Hassan F, Shaaban J. Use of traditional/complimentary medicine (T/ CM) among adult patients attending Family Medicine Clinic at Hospital Universiti Sains Malaysia. Int Med J 2005;12:133-8.

2. Hasan SS, Ahmed SI, Bukhari NI, Loon WC. Use of complementary and alternative medicine among patients with chronic diseases at outpatient clinics. Complement Ther Clin Pract 2009;15:152-7.

3. Ismail Z, Mohamed R, Mohd Hassan MH, Wan Su K. Usage of traditional medicines among elderly and the prevalence of prednisolone contamination. Malays J Med Sci 2005;12:50-5.

4. Bhattacharyya A, Kaushal K, Tymms DJ, Davis JR. Steroid withdrawal syndrome after successful treatment of Cushing's syndrome: a reminder. Eur J Endocrinol 2005;153:207-10.

5. Ernst E. Toxic heavy metals and undeclared drugs in Asian herbal medicines. Trends Pharmacol Sci 2002;23:136-9.

6. Krapf R. Development of Cushing's syndrome after use of a herbal remedy. Lancet 2002;360:1884.

7. Oldenburg-Ligtenberg PC, van der Westerlaken MM. A woman with Cushing's syndrome after use of an Indonesian herb: a case report. Neth J Med 2007;65:150-2.

8. Sazlina S, Zaiton A. Cushing's syndrome secondary to adulterated complementary and alternative medicine. Malays Fam Physician 2009;4:94-7.

9. Hopkins RL, Leinung MC. Exogenous Cushing's syndrome and glucocorticoid withdrawal. Endocrinol Metab Clin North Am 2005;34:37184.

10. Iliopoulou A, Abbas A, Murray R. How to manage withdrawal of glucocorticoid therapy. Prescriber 2013;24:23-9. 